\title{
Research On Small Signal Magnify-Chain Circuit Based On Pressure Bridge
}

\author{
Huang Jian, Fan Hui \\ XiJing University ,Xi'an,710123,China \\ 565200245@qq.com
}

Keywords: small signal; meter amplifier; auto-return-to-zero technology; common mode rejection ratio

\begin{abstract}
Weak small signal acquisition is particularly important in electronic design and data acquisition. Effective processing of small signals must be magnified, while magnifying small signals can produce interference magnification and cause errors. In order to solve the interference and detune in small signal magnification, based on the scrutiny of small signal features, this paper suggests the adoption of precision meter amplifier and auto-return-to-zero technology to magnify the weak signal. The precision meter amplifier can realize the higher common mode rejection ratio, and the auto-return-to-zero technology can reduce the detune errors. Accordingly, the both combination can realize the stable and effective magnification of 110 times of the weak signals. The test results indicated that under TINA-TI, the simulation was carried out so that the millivolt grade signal could be magnified to several volts steadily and the interference and errors can be suppressed effectively. This design is of certain practicality and reference values, and provides a kind of new thinking and designing method for studying small signal magnifying chain circuit.
\end{abstract}

\section{Introduction}

In order to realize the detection of non-electric quantity signals, people have always adopted

the transducers of various types to convert them into the weak current or the high voltage signals, and how do we carry out the acquisition and processing of these weak electric signals? At present, the usually-used method is first to magnify the signals, and then to filter the waves. The simulation voltage values after filtering waves are connected into $\mathrm{A} / \mathrm{D}$ acquisition electric circuit to convert them into the digital signals; and then, they are fed into the processor for their processing. In this data chain circuit, signal magnification and filtering waves are obviously special important. If the data are instable or the interference are not be effectively filtered, the voltage values fed into A/D will produce large fluctuation, whereby the acquisition effects will be seriously affected. As to some low frequency signals, for example, the signals transmitted by the pressure transducer can effectively eliminate the interferences ${ }^{[1-4]}$. If they are magnified using the precision meter amplifier and through the input voltage value correlative subtract, and the detune errors can be reduced through the auto-return-to-zero operation with high precision, whereby making it output the stable voltage values into A/D conversion chip. Accordingly, the acquisition and processing of small signal voltage or electric current signals can be realized.

\section{System Scheme Design}

The system frame chant is shown in Fig 1. Small signal transducer electric circuit may be the pressure bridge transducer, supersonic transducer, etc. various types of transducers. The meter amplifier has adopted the high precision meter amplifier INA128 made by TI company. When the input signal is $200 \mathrm{KHz}$, the gain $\mathrm{G}$ can reach 100, whose common mode suppression ratio CMRR reaches $120 \mathrm{dbs}$, being able to suppress the interference signals effectively. The electric circuit produced by negative pressure is $-3.6 \mathrm{~V}$ power supply voltage provided by INA128. In electric circuit design, INA128 can magnify the input signals to 11 times as much. Auto-return-to-zero amplifier has adopted OPA334 ${ }^{[5]}$ made by TI Company. The temporary-Wave-Steady-Zero Technology makes the input detune voltage be the maximum $5 \mu \mathrm{V}$, and the maximum detune voltage 
drift is $0.55 \mu \mathrm{V} /{ }^{\circ} \mathrm{C}$, which is the detune voltage produced by the effective suppression meter amplifier. The signals input by INA128 are magnified by 10 times again. The total realizations of two grade magnification to the small signal magnification reach to 110 times, and then, the small signals are fed into the high precision 16-bit A/D Conversion Chip ADS1146, ADS1146 adopts the well-regulated Voltage ${ }^{[6]}$.

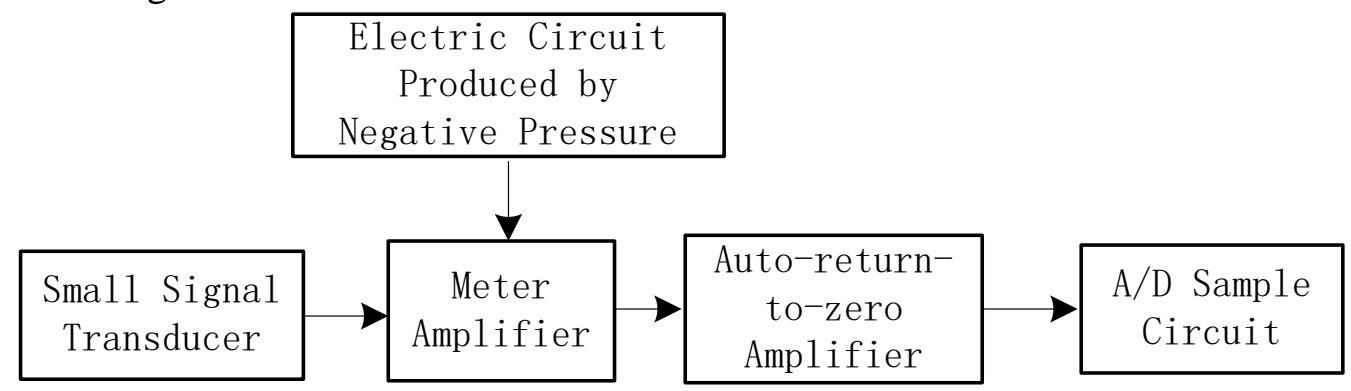

Fig. 1 System Design Frame Chart

\section{Detail Design and Key Technology}

\section{Pressure Bridge and Meter Magnification Electric Circuit Design}

Pressure Bridge and meter magnification electric circuit are shown in Fig 2 Pressure Bridge

Electric circuit consists of three $10 \mathrm{~K} \Omega$ resistances and one $10 \mathrm{~K} \Omega$ potentiometer. The meter amplifier adopts INA128; two-circuit voltages of Pressure Bridge are fed into IN+ and IN- of INA128. Appropriate regulation of potentiometer is able to change the potential difference of IN+ and IN-. The negative voltage is produced by TPS60400. The 2-foot input $+3.6 \mathrm{~V}$ of TPS60400 can be converted via the inner voltage into the one-foot output $-3.6 \mathrm{~V}$ voltage ${ }^{[7]}$, to supply the 4-foot $\mathrm{V}$ end of INA128 so as to realize the double power supply to INA128. The magnification of INA128 is shown in equation (1):

$$
\mathrm{G}=1+\frac{50 \mathrm{~K}}{\mathrm{R}_{\mathrm{g}}}
$$

As to the electric circuit diagram indicated in Fig. 2, when $\mathrm{Rg}=5 \mathrm{~K} \Omega, \mathrm{G}=11$,appropriately-regulated $\mathrm{Rg}$ value can change the arithmetic amplifier gains. When $\mathrm{Rg}=1 \mathrm{~K} \Omega, \mathrm{G}=51, \mathrm{Rg}=500 \Omega, \mathrm{G}=101, \mathrm{G}$ maximum value in theory can reach 1000 . Regulating resistance R1 value in electric circuit of pressure bridge transducer can change the input end voltage of the IN+ end of the input INA128 ${ }^{[8]}$, whose concrete calculation can refer to equation(2):

$$
V_{0}=\left(V_{1}-V_{2}\right) \times G
$$

In analyzing equation(2), when we input voltage to IN+ and IN-, the interference signals are always input into running input end. Assuming that the interference signal is Vn, it interferes in IN+ and at the same , it would also interfere in IN-. When there is interference, the input into INA128 3 -foot voltage is $\mathrm{V} 1+\mathrm{V}_{\mathrm{N}}$; and the input into INA128 2-foot voltage is $\mathrm{V} 2+\mathrm{V}_{\mathrm{N}}$, because INA128 is the differential arithmetic amplifier. When equation(2) is used I calculation of $\left(\mathrm{V} 1+\mathrm{V}_{\mathrm{N}}\right)-$ $\left(\mathrm{V} 2+\mathrm{V}_{\mathrm{N}}\right)=(\mathrm{V} 1-\mathrm{V} 2)$, it is just good to eliminate the interference signals. When resistance value of pressure bridge potentiometer $\mathrm{R} 1$ is regulated to $9.9 \mathrm{~K} \Omega$, calculation can be carried out to obtain $\mathrm{V} 1=(10 / 9.9+10) * 3.6 \mathrm{~V}=1.809 \mathrm{~V}$ in terms of series resistance partial pressure. After R2 and R4 series resistance partial pressure, calculation is performed to obtain $\mathrm{V} 2=1.8 \mathrm{~V}$. thus, substituting it into equation(2), the calculation can be performed to obtain $\mathrm{V}_{\mathrm{O}}=(\mathrm{V} 1-\mathrm{V} 2) * 11+1.8 \mathrm{~V}=(1.809 \mathrm{~V}-1.8 \mathrm{~V}) * 11=0.099 \mathrm{~V}=99 \mathrm{mV}$. Accordingly, it can be seen that this electric circuit will be input as $9 \mathrm{mV}$ signal being magnified by 11 times as much, whereby becoming $99 \mathrm{mV}^{[9]}$. Appropriate regulation of $\mathrm{R} 1$ resistance value can obtain the output results of differences. Attention must be paid to INA128, whose maximum output voltage is 3.6V. in terms of equation (2), the inverse derivation can be performed to obtain V1 and V2 maximum voltage differences which must be less than $3.6 \mathrm{~V} / 11=0.3272 \mathrm{~V}$. 


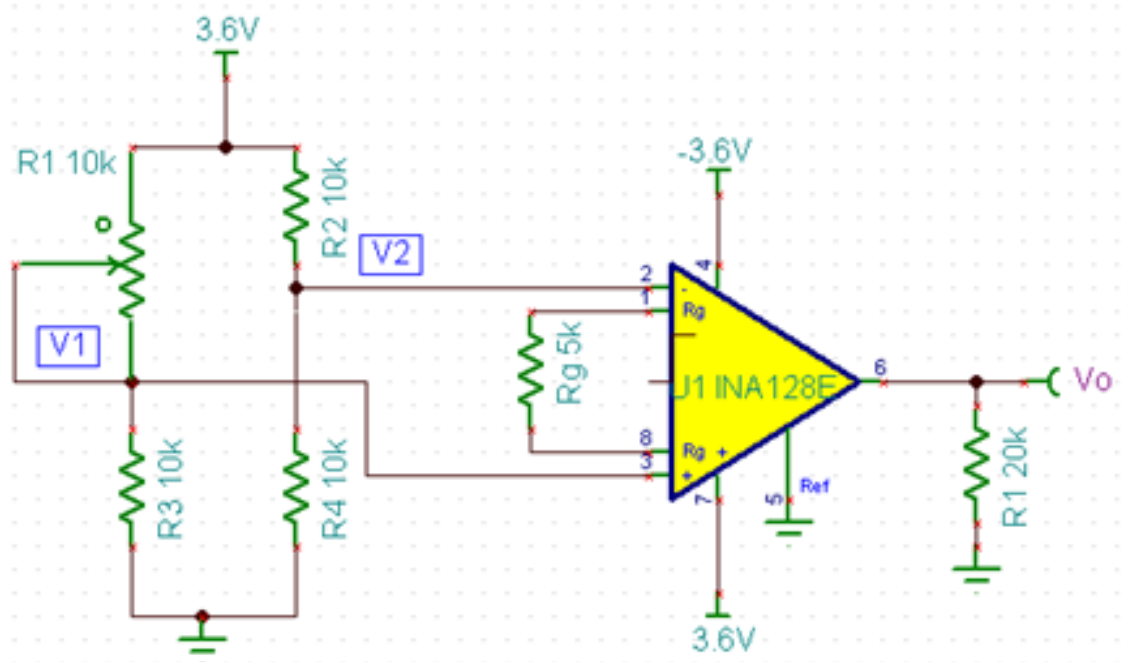

Fig.2 Amplified Electric Circuit Diagram of Pressure Bridge and Meter Amplifier

\section{A/D Sample Electric Circuit Design}

The 16 bit high-accuracy A/D Chips ADS1146 is adopted. The conversion voltage Vo2 is joined to 7-foot ADS1146. The converted digital quantity is output with SPI bus line pattern via 14 and 15 feet, which is convenient to join to the micro-processors of various kinds. Voltage adopts $3.6 \mathrm{~V}$ to provide electricity. The minimum resolution reached by 16 bit $\mathrm{A} / \mathrm{D}$ can be $3.6 \mathrm{~V} / 65536=0.05 \mathrm{mV}$, being sufficient to satisfy the requirements.

Test results

Based on the above hardware design, as shown in Fig.3, and the simulation can be carried out under TINA-TI.

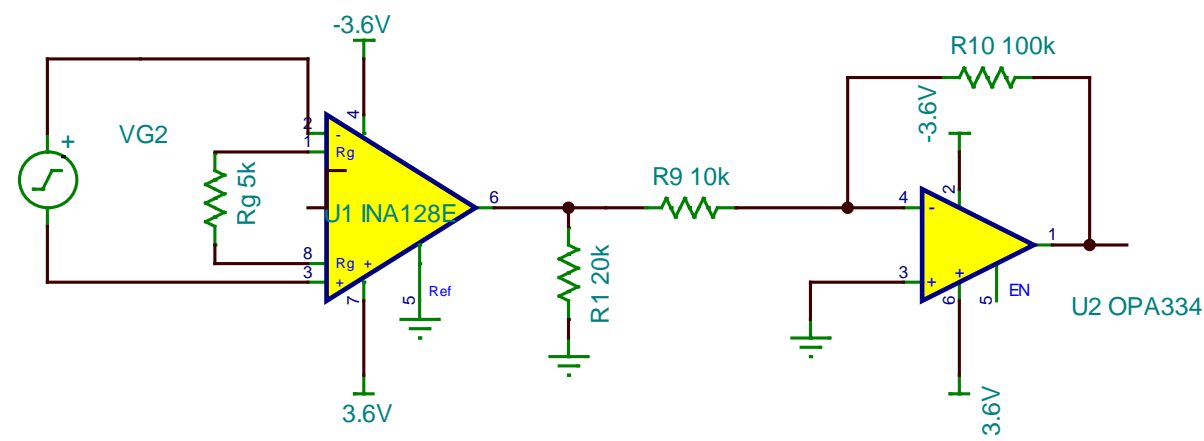

Fig.3. Simulation Connection Diagram

The input frequency is $50 \mathrm{~Hz}$, and the amplitude is the $0 \sim 20 \mathrm{mv}$ sinusoidal wave, and the output wave pattern after being magnified by 110 times as much is shown in Fig.4. 


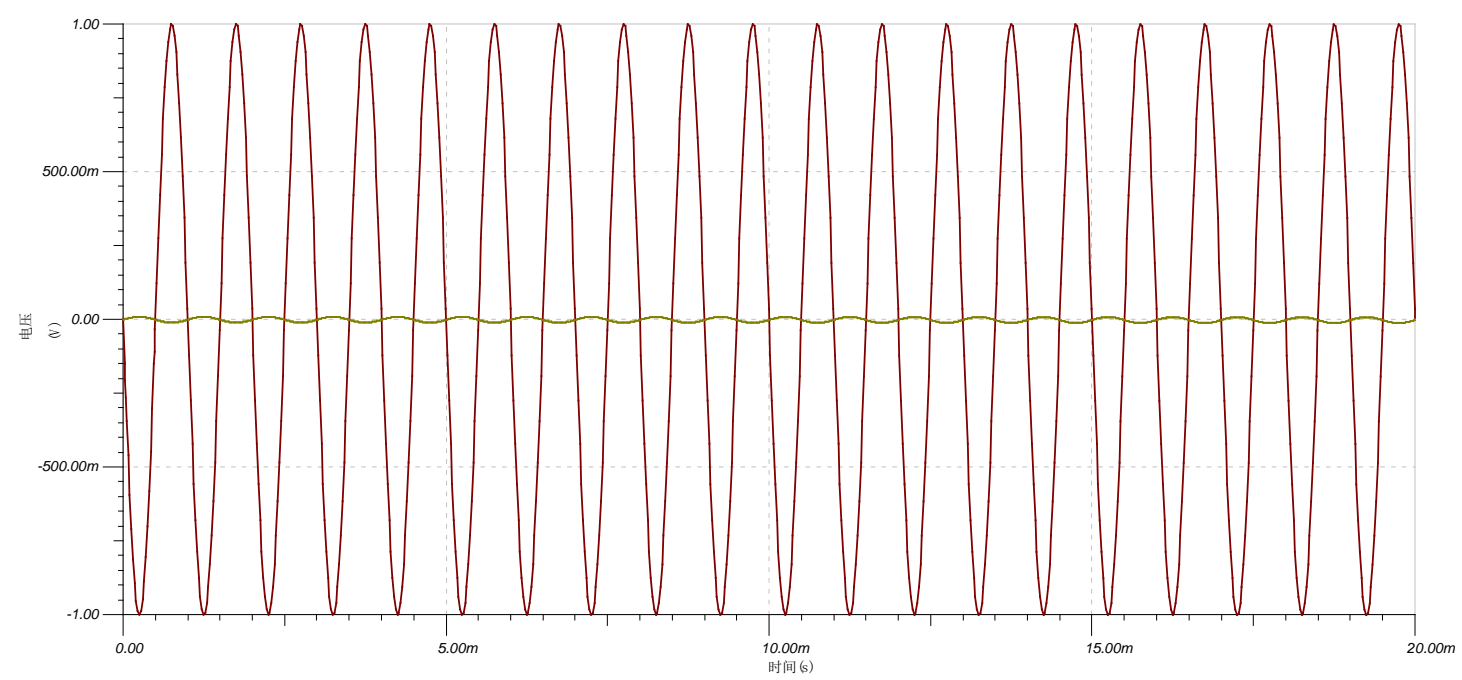

Fig.4 Small Signal Magnified Diagram

\section{Conclusions}

This suggests a kind of small signal magnification data chain circuit using the precision instrumentation amplifier and the auto-return-to-zero precision amplifier. The adaptation of differential magnification electric circuit can omit the design of wave filter electric circuit. Based on the principle, the hardware electric circuit is set up, and the simulation is carried out under TINA-TI. The simulation results indicate that INA128 is able to magnify the weak signals by 11 times as much effectively, which is joined to OPA334 after, to magnify 0 20mv sinusoidal wave to 110 times effectively and to be convenient for A/D electric circuit acquisition processing. Appropriative regulation of hardware electric circuit parameters or selection of different devices can design the acquisition chain circuit to satisfy the special demand for small signals ${ }^{[10]}$. This chain circuit can be used in the signal acquisitions of pressure, supersonics and sounds, etc. and be of a certain reference and practical values.

\section{References}

[1] Texas Instruments. OPA334(M). Shanghai,2003.9

[2] Texas Instruments. INA128(M). Shanghai,2003.9

[3] Texas Instruments. TINA-TI Fast Guide to Gateway (M). Shanghai,2003.9

[4] HePing.etal. RBF Network Model for Pressure Transducer Temperature Shift Compensation[J] Journal of Instruments and Meters. 2008,29(3):572-576

[5] Lu Haojie etal. The Design and Thermal Analysis of High-Performances MEMS Capacity Pressure Transducer [J], Optical Precision Engineering,2010,18(5)1166-1173.

[6]Zhang XiaoLi etal. Research Existing Conditions and Development of Hot-tolerance pressure Transducers[J]. Transducers and Micro-system,2011.30(2):1-4.

[7]Hou Peigou etal. Research and Design of micro-work Consumption Wireless Pressure Transmitter Based on MSP430[J] Meter Technology and Transducer,2011,2(2):19-25

[8] Zhang Jiajun etal. High-Accuracy Pressure Transmitter of Single Chip Based on MSP430 [J].Meter Technology and Transducer,2010,1:32-35 